\title{
Renal Artery Stenosis in Patients with Peripheral Vascular Disease in Kuwait
}

\author{
A. Ahmed ${ }^{a}$ M.R.N. Nampoory ${ }^{b} \quad$ M. Sheikh ${ }^{a} \quad$ K.V. Johny \\ Departments of ${ }^{a}$ Radiology and ${ }^{b}$ Medicine, Faculty of Medicine, University of Kuwait, and \\ Mubarak Al-Kabeer Hospital, Kuwait
}

\section{Key Words}

Atherosclerosis - Peripheral vascular disease •

Renal artery stenosis

\begin{abstract}
Objective: The aim of this study was to identify the incidence of atherosclerotic renal artery stenosis (RAS) in patients with peripheral vascular disease (PVD) and its relation to any known risk factors. Subjects and Methods: This prospective study was conducted on 212 patients who were subjected to peripheral angiography for symptoms of PVD over a 3-year period from 1995 to 1998 at the Mubarak Al-Kabeer Hospital, Kuwait. Angiographic evidence of atherosclerotic disease and its severity was recorded in renal, abdominal aorta, iliac, femoral, popliteal and below-knee arteries. In addition, a detailed search of identifiable risk factors was done using history, clinical examination and laboratory studies. Results: The incidence of significant atherosclerotic RAS (more than $50 \%$ diameter stenosis) in patients with PVD was $15 / 212$ $(7.07 \%)$ with no significant difference in ratio between males and females $(p=0.3)$ compared to that of PVD alone. Patients with common iliac and femoral artery lesions had a high incidence of RAS $(93.3$ and $86.7 \%$, respectively) with more than $80 \%$ probability in RAS patients with involvement of these vessels. There was significant renal impairment $(p<0.005)$, as assessed by
\end{abstract}

serum creatinine levels, in patients with RAS compared to those who did not have it. There was a high incidence of smoking in patients with RAS $(p=0.02)$, and smoking was the only risk factor identified in these subjects. Conclusions: Patients with iliac or femoral atherosclerotic disease have a high probability of associated RAS. Presence of renal impairment in patients with PVD is highly indicative of RAS. Smoking is the only identified risk factor for RAS in association with PVD in our population.

Copyright $@ 2005$ S. Karger AG, Basel

\section{Introduction}

Atherosclerotic renal artery stenosis (RAS) is a recognized cause of secondary hypertension and chronic renal failure [1]. It has been estimated that this condition may account for up to $20 \%$ of the end-stage renal failure in patients over 50 years old [1]. Chronic renal failure due to renal artery stenosis (RAS) is a potentially reversible disorder. Various studies [2-5] including ours [6] have shown that corrective procedures like surgery and percutaneous angioplasty are effective in correcting the stenosis and preserving renal function. RAS, on the other hand, can be present without hypertension [7]. Hence progressive RAS can occur without hypertension leading to progressive renal failure especially in the elderly population [3]. Therefore, early recognition of RAS is important be-

\section{KARGER}

Fax +4161306 1234

E-Mail karger@karger.ch

www.karger.com
C 2005 S. Karger AG, Basel

$1011-7571 / 05 / 0146-0386 \$ 22.00 / 0$

Accessible online at:

www.karger.com/mpp
Dr. Adel Ahmed

Department of Radiology, Faculty of Medicine

Kuwait University, P.O. Box 24923

Safat Code 13110 (Kuwait)

Tel. +965 7888493, Fax +9655330473, E-Mail adelaaa@hotmail.com 
cause of its progressive nature and the risk of precipitating an acute deterioration in renal function, especially in asymptomatic patients. The incidence of RAS increases with age and is higher in subjects with atherosclerotic disease in other vessels $[1,6,8,9]$. The literature on the incidence and prediction of RAS, especially in patients with atherosclerotic arterial disease elsewhere are scanty. Only few reports from the western countries are available based on post-mortem series and angiographic series [6,9-11]. This prospective study was conducted to determine the incidence of RAS in patients with peripheral arterial disease and to identify any associated high risk factors.

\section{Materials and Methods}

Two hundred and twelve patients (163 males and 49 females) who were referred for peripheral angiography at Mubarak Al-Kabeer Hospital, Kuwait, from 1995 to 1998, were the subjects of this study. Detailed medical history and clinical examination were obtained from all patients. Special attention was given to presence and grade of claudication, presence of critical ischemia (rest pain, ulcers and gangrene), impotence, elevated blood pressure, diabetes, hypercholesterolemia, increased level of triglycerides and smoking. Smokers were defined as those who were currently or previously regular tobacco smokers. All the patients with known hypertension were on antihypertensive medication. None of the patients were on antihyperlipidemic treatment except those with known coronary heart disease.

Angiography was performed via right common femoral approach using Seldinger technique. Digital subtraction angiography was obtained in all patients. The following views were acquired: anteroposterior view of abdominal aorta/renal arteries, 12 degrees left or right oblique views of renal and iliac arteries, anteroposterior views of superficial femoral arteries, popliteal and below-knee arteries, (peroneal, anterior and posterior tibial arteries).

Patients found to have vascular irregularity resulting in various stenoses or complete occlusion were deemed to have the vascular disease. Regarding renal vessels, patients who were found to have more than $50 \%$ narrowing in diameter were considered to have significant RAS. Angiography was performed after obtaining informed consent from the patients. The level and extent of disease in the peripheral vessels and the renal vessels were recorded. Thirty age- and sex-matched controls without RAS but with peripheral vascular disease (PVD) were included for comparison with those with RAS. Relevant clinical information was also collected as described previously.

\section{Results}

Of the 212 patients, 15 (10 males and 5 females) were found to have RAS with an incidence of $7.07 \%$. The incidence was not different in either sexes $(p=0.34)$. For each of the 15 patients with RAS, two controls were se-
Table 1. Comparison of the predictors/risk factors for atherosclerotic renal disease

\begin{tabular}{llll}
\hline Diseases & $\begin{array}{l}\text { With RAS } \\
(\mathrm{n}=15)\end{array}$ & $\begin{array}{l}\text { Without RAS } \\
(\mathrm{n}=30)\end{array}$ & $\mathrm{p}$ value \\
\hline Diabetes mellitus & $7(46.6)$ & $23(76.0)$ & 0.9981 \\
Coronary artery disease & $6(40.0)$ & $14(46.6)$ & 0.7569 \\
Smoking & $9(60.0)$ & $7(23.3)$ & 0.0227 \\
Hypertension & $4(26.7)$ & $8(26.7)$ & 1.0 \\
\hline
\end{tabular}

Figures in parentheses are percentages.

Table 2. RAS in relation to other peripheral vascular disease

\begin{tabular}{lrcl}
\hline Vessels involved & $\begin{array}{l}\text { With RAS } \\
(\mathrm{n}=15)\end{array}$ & $\begin{array}{l}\text { Without RAS } \\
(\mathrm{n}=197)\end{array}$ & $\mathrm{p}$ value \\
\hline Aorta & $10(66.7)$ & $50(25.4)$ & 0.0017 \\
Common iliac & $14(93.3)$ & $77(39.1)$ & 0.0001 \\
External iliac & $5(33.3)$ & $11(5.6)$ & 0.0028 \\
Femoral & $13(86.7)$ & $111(56.3)$ & 0.0273 \\
Popliteal & $2(13.3)$ & $40(20.3)$ & 0.7394 \\
Below knee & $6(40.0)$ & $78(39.6)$ & 1.0 \\
\hline
\end{tabular}

Figures in parentheses are percentages.

lected from the cohort with PVD without RAS for comparison. In this group, information collected was similar to that collected for the group with RAS. Thirty ageand sex-matched controls without RAS but with PVD were included for comparison with those with RAS. Table 1 shows comparison of predictor/risk factors for RAS between the two groups. The prevalence of diabetes mellitus, coronary artery disease, hypertension and hyperlipidemia was similar in both groups, but smoking was found to be a significant $(\mathrm{p}=0.02)$ risk factor predictor for RAS in subjects with PVD. The incidence of RAS in relation to other peripheral vascular involvement is shown in table 2. Atherosclerotic occlusive disease of aorta $(\mathrm{p}=$ $0.001)$, common iliac $(p=0.0001)$, femoral $(p=0.027)$ and external iliac $(\mathrm{p}=0.002)$ significantly correlated with RAS. Systolic (149.9 \pm 86 and $143.5 \pm 24 \mathrm{~mm} \mathrm{Hg})$ and diastolic $(96.6 \pm 10.8$ and $94 \pm 10.5 \mathrm{~mm} \mathrm{Hg})$ blood pressures were not significantly ( $p=0.43$ and 0.49 ) different between subjects with and without RAS (table 3). Biochemical parameters related to RAS are shown in table 4. Blood urea, serum cholesterol and triglyceride levels were similar in both groups. Renal function, as assessed 
Table 3. Difference in blood pressure between patients with and without RAS

\begin{tabular}{|c|c|c|c|}
\hline Parameter & $\begin{array}{l}\text { With RAS } \\
(\mathrm{n}=15)\end{array}$ & $\begin{array}{l}\text { Without RAS } \\
(\mathrm{n}=30)\end{array}$ & $\mathrm{p}$ \\
\hline Systolic, mm Hg & $149.9 \pm 86.6$ & $143.5 \pm 24$ & 0.43 \\
\hline Diastolic, $\mathrm{mm} \mathrm{Hg}$ & $96.6 \pm 10.8$ & $94 \pm 10.5$ & 0.49 \\
\hline
\end{tabular}

Values are expressed as mean $\pm \mathrm{SD}$.

Table 4. Biochemical parameters in patients with and without RAS

\begin{tabular}{lccl}
\hline Parameter & $\begin{array}{l}\text { With RAS } \\
(\mathrm{n}=15)\end{array}$ & $\begin{array}{l}\text { Without RAS } \\
(\mathrm{n}=30)\end{array}$ & $\mathrm{p}$ \\
\hline Urea, mmol/1 & $8.2 \pm 4.2$ & $6.93 \pm 3.3$ & 0.3 \\
Creatinine, $\mu \mathrm{mol} / \mathrm{l}$ & $173.2 \pm 126.8$ & $94 \pm 29.2$ & 0.004 \\
Cholesterol, $\mathrm{mmol} / \mathrm{l}$ & $6.7 \pm 1.9$ & $6.1 \pm 1.3$ & 0.68 \\
Triglycerides, $\mathrm{mmol} / 1$ & $2.02 \pm 0.24$ & $3.5 \pm 1.5$ & 0.24 \\
\hline
\end{tabular}

Values are expressed as mean $\pm \mathrm{SD}$.

by mean creatinine, was significantly $(\mathrm{p}=0.004)$ worse in subjects with RAS $(173.2 \pm 126.8 \mu \mathrm{m} / \mathrm{l})$ compared to those without RAS $(94 \pm 29.2 \mu \mathrm{m} / \mathrm{l})$.

\section{Discussion}

Incidence of RAS in subjects with ESRD is variably reported from 1.7 to $22 \%[6,9]$. Ischemic renal disease (IRD) is the etiology for ESRD in such patients. Atherosclerosis is the most common cause of IRD, reported to account for $60-97 \%$ of renal arterial lesions [12]. In a recent report on 386 patients with PVD, 33\% had significant $(>50 \%)$ RAS [10]. Similar incidences were reported earlier [13]. Our incidence of $7.7 \%$ is lower than in other reports in the literature. It has been shown that RAS is more commonly associated with PVD than coronary artery disease, which is reported to be only $15 \%$ in 1,235 patients undergoing coronary angiography [10]. Similar to our earlier report in subjects with IRD, we found low incidence of RAS amongst our patients with PVD [6].

Renal failure, proteinuria, diabetes mellitus, coronary artery disease, hypertension, hyperlipidemia and smoking as predictor/risk factors for RAS and IRD in elderly males have been a subject of discussion in various studies
$[3,14,15]$. We found renal function at time of presentation with IRD was worse in subjects with ESRD [6]. Similarly, present data also showed significant renal impairment in subjects with PVD with RAS compared to those without RAS. Hence our observation denotes that renal impairment with PVD could predict presence of RAS. We could not find any other specific predictors/risk factors for RAS. There are available data showing high incidence of RAS in subjects with coronary artery disease $[10,16]$. High incidence of atherosclerosis in diabetics, hyperlipidemics and hypertensives could justifiably lead to RAS. But in this study we found similar incidence of diabetes mellitus, hyperlipidemia and coronary artery disease in PVD subjects with and without RAS. Prevalence of subjects smoking tobacco is high in Kuwait [17]. Tobacco smoking is known to predispose to occlusive vascular disease, especially in diseases like thromboangiitis obliterans. We observed significantly higher prevalence of people smoking tobacco among our patients with PVD and RAS than those without RAS. Evidently, smoking could be a predictor/risk factor for RAS in subjects with PVD.

In a retrospective study, Metcalfe et al. [8] reported high incidence of RAS (36.2\%) in patients with PVD. They observed that the incidence of RAS was high in subjects with femoral artery lesions (42.1\%) compared to those who did not have it (9.7\%). To our knowledge there are no other similar reports in English literature. Present data showed significantly high prevalence of RAS when subjects had femoral and iliac atherosclerotic lesions either unilateral or bilateral.

\section{Conclusion}

In conclusion, patients with iliac and femoral atherosclerotic disease have higher significant probability of having RAS. Presence of renal impairment in subjects with PVD is highly indicative of RAS. Smoking is the only identifiable risk factor for RAS in association with PVD in our patient population. 


\section{References}

1 Mailloux LU, Napolitano B, Belkicci AG, et al: Renal vascular disease causing end-stage renal disease, incidence, clinical correlates and outcomes: a 20-year clinical experience. Am J Kidney Dis 1994;24:622-629.

-2 Rimmer JM, Gennari FJ: Atherosclerotic renovascular disease and progressive renal failure. Ann Intern Med 1993;118:712-719.

$\checkmark 3$ Jacobson HR: Ischemic renal disease. An overlooked clinical entity? Kidney Int 1988;34: 729-743.

$\checkmark 4$ Libertino JA, Bosco PJ, Ying CY, et al: Renal revascularization to preserve and restore renal function. J Urol 1992;147:1485-1487.

$\checkmark 5$ Hansen KJ, Starr SM, Sands RE, et al: Contemporary surgical management of renovascular disease. J Vasc Surg 1992;16:319-331.

-6 Sheikh M, Nampoory MRN, Sinan T, et al: Ischemic renal disease in Kuwait. Angiology 2002;53:83-88.
7 Kimberley JH: Prevalence of ischemic nephropathy in the atherosclerotic population. Am J Kidney Dis 1994;24:615-621.

8 Metcalfe W, Reid AW, Geddes CC: Prevalence of angiographic atherosclerotic renal artery disease and its relationship to the anatomical extent of peripheral vascular atherosclerosis. Nephrol Dial Transplant 1999; 14:105-108.

$\checkmark 9$ Appel RG, Bleyer AJ, Reavis S, et al: Renovascular disease in older patients beginning renal replacement therapy. Kidney Int 1995;48: 171-176.

10 Uzu T, Inoue T, Fujii T, et al: Prevalence and predictors of renal artery stenosis in patients with myocardial infarction. Am J Kidney Dis 1997;29:733-738.

11 Trude CL, Peter MTP, Annette VDB: Incidental renal artery stenosis in peripheral vascular disease: a case for treatment? Kidney Int 2001; 59:1480-1483.

12 Greco BA, Breyer JA: Atherosclerotic ischemic renal disease. Am J Kidney Dis 1997;29:167187.
13 Bryan NB, Jon SO, Yolanda TB, et al: Peripheral vascular disease and renal transplant artery stenosis: a reappraisal of transplant renovascular disease. Clin Transplant 1999;13: 349-355.

14 Abuelo JG: Diagnosing vascular cause of renal failure. Ann Intern Med 1995;123:601-614.

15 Meyrier A, Buchet P, Simon P, et al: Atheromatous renal disease. Am J Med 1988;85:139_ 146.

16 Jean WJ, Al-Bitar I, Zwicke DL, et al: High incidence of renal artery stenosis in patients with coronary artery disease. Cathet Cardiovasc Diagn 1994;32:8-10.

17 Memon A, Moody PM, Sugathan TN, et al: Epidemiology of smoking among Kuwaiti adults: prevalence, characteristics and attitudes. Bull World Health Organ 2000;78: 1306-1315. 
Filename: mpp729

Article-No: 729, Fig.: 0, Tab.: 4

$<$ Issueid $>006<$ /Issueid $>$

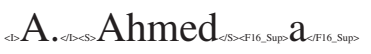

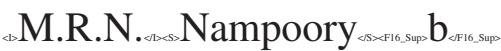

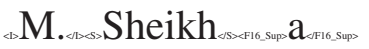

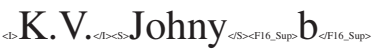

Sorry, there is no abstract. Read the first few lines of the text instead! 